\title{
Control-Oriented Model of a Generating Set comprising a Diesel Engine and a Synchronous Generator
}

\author{
M. Tuffaha J. T. Gravdahl \\ Department of Engineering Cybernetics, Norwegian University of Science and Technology, N-7491 Trondheim, \\ Norway. E-mail: \{Mutaz.Tuffaha,Jan. Tommy.Gravdahl\} @itk.ntnu.no
}

\begin{abstract}
A generating set (Genset) comprises a prime mover such as a Diesel Engine, and a synchronous generator. The most important controllers of such systems are the speed governor to regulate the engine or shaft speed and the automatic voltage regulator (AVR) to regulate the terminal voltage. The speed governor is a PID controller that uses the difference between the speed and its desired value as a feedback signal to change the fuel mass input by changing the fuel rack position. AVR is also a PID that uses the difference between the terminal voltage of the generator and its desired value, and changes it by manipulating the voltage of the field excitation circuit. Thus, the two controllers act separately. That is to say, if the speed varies from the desired value, the speed governor will react, while the AVR will not react as long as the voltage is stable, and vice versa. In this work, a control-oriented model is suggested for a Genset, and then a controller, that regulates the shaft speed and the terminal voltage, is designed by feedback linearisation. The proposed controller has two inputs: the fuel mass and the field circuit voltage. Simulations show that the proposed controller makes the two inputs act, simultaneously. Thus, any change of the speed e.g., forces the two input controls to react, in contrast to the ordinary PID controllers. Further, we discuss the robustness of the proposed controller to uncertainties and time delay.
\end{abstract}

Keywords: Genset, Diesel Engine, Synchronous Generators

\section{Introduction}

The most important control objectives in power systems stability studies are the voltage control, which leads to reactive power control, and frequency control, which leads to active power control. For these purposes, the automatic voltage regulator (AVR) and speed governor are still the corner stone in the control hierarchy. The AVR is a PID controller that uses the error between the terminal voltage and its desired value as a feedback signal to control the terminal voltage by controlling the magnetic field of the rotor, which can be produced by an excitation circuit or a permanent magnet, as will be explained later. The speed gover- nor, on the other hand, regulates the torque provided by the prime mover, and thus the rotational speed, on which the frequency of the produced currents and voltages depends. The manipulated control inputs of both controllers depend on the type of the machine and the prime mover used.

For large power systems that contain many generating units this looks sufficient, because the complicated hierarchical control structure of power systems may not be so vulnerable to small variations in voltage or frequency. However, for isolated power systems this may not be the case. McCowan et al. (2003) showed that the speed governor and the AVR act separately, which may create problems. Then, they gave the following 
example to show their point. If the load increases suddenly, the speed of the engine and the terminal voltage will drop. Hence, both controllers will react by increasing the fuel input to the engine, and the field circuit excitation. However, this may cause the terminal voltage to increase above the steady-state value, and hence increasing the load on the engine, which in turn drives the governor to increase the fuel, and stability may be lost or retarded, McCowan et al. (2003). That is why, the researchers in this field have been trying to design a controller that drives the two control inputs; the field excitation and the fuel input, simultaneously, since it is believed that if the two input controls are coordinated, the performance of the controller under peculiar situations such as the one explained in McCowan et al. (2003) will be improved.

Rahman et al. (1996) proposed a non-linear model of the isolated permanent magnet synchronous generator driven by a Diesel engine. Then, the non-linear model was linearised around steady-state values. Further, a performance index was suggested to find an optimal controller to regulate the terminal voltage and frequency, where they used two control inputs; the fuel mass to control the torque of the engine, and the firing angle of a thyristor to control the terminal voltage, Rahman et al. (1996). Although Rahman et al. (1996) used a first order model of the Diesel engine, the simulations presented showed the effectiveness of the proposed controller. Then, Goh et al. (2003) proposed a sliding-mode speed controller of the Genset. Later, McGowan et al. (2006) proposed a fuzzy logic speed controller. Recently, the phenomenon of power oscillations of marine power systems, that have several Gensets working in parallel, was studied and analysed in Huang (2012). Moreover, the author proposed a non-linear model of the Genset and designed a robust synthetic controller to control this phenomenon, Huang (2012). In spite of the novelty of his proposed controller, Huang (2012) did not propose a model for the engine torque, instead he used the actuator dynamics. Further, the model proposed for the dynamics of the terminal voltage may not be adequate for such purposes.

The current authors proposed a model for the Genset that has a propeller in addition to the engine and the machine in Tuffaha and Gravdahl (2014). However, Tuffaha and Gravdahl (2014) used the flux linkage as a state variable which is usually difficult to be measured. Besides, they used the first-order model of the Diesel engine in Fossen (1994) that uses the fuel rack input to manipulate the torque, without taking in consideration the air dynamics. In this work, the terminal voltage is used instead of the flux linkage because the terminal voltage can be measured more easily than the flux linkage. Further, the model of the torque developed by the Diesel engine proposed by Jensen et al. (1991) is used here because it describes the torque more accurately than the first-order model in Fossen (1994), and it takes the air dynamics in consideration, as will be shown later. Then, a controller is designed by using feedback linearisation. The proposed controller has two manipulated control inputs; the fuel mass input to the engine, and the field excitation circuit voltage. The air/fuel ratio in the Diesel engine is modelled as uncertain parameter. The simulations presented, show that the two control inputs act simultaneously, i.e. they communicate with each other, as will be explained in the discussion of the simulation results. In addition, the simulations show that, the controller performs satisfactorily for small values of time delay imposed by the Diesel engine, when it is considered. Moreover, our simulations show that the proposed controller can be considered robust to the uncertainty of the air/fuel ratio.

\section{Mathematical Model}

In this section, we present the proposed model of the Genset and its controller.

\subsection{Synchronous Machine}

Synchronous machines are essential components of power systems. They can be used as generators or as motors. The basic principle of operation of the synchronous machine as a generator can be explained as follows. A synchronous machine comprises a rotating part called rotor, and a stationary part called stator. The stator carries the armature windings which are 3phase ac windings separated by $120^{\circ}$. On the other hand, the rotor provides a direct magnetic field by either a permanent magnet or a dc field winding around the rotor powered by an exciter which may take several forms. When the rotor is rotated by a prime mover such as a Diesel engine in our case, an electromotive force (emf) will be induced in the armature windings. Because of the windings structure, an ac current will be induced in the stator, and hence an ac magnetic field will be created from each phase winding in the armature. Thus, the resultant magnetic field of the armature windings will be rotating, i.e. sinusoidal function of time. The rotating magnetic field from the stator tries to catch the rotating magnetic field produced from the rotor, and hence a torque is created and transformed into electrical energy in the form of the ac produced in the stator. The steady state speed with which the magnetic field produced in the armature rotates is called the synchronous speed. It can be shown that this 
synchronous speed is related to the mechanical speed of the rotor through:

$$
\Omega_{M}=\frac{2}{p} \Omega_{S}
$$

where $p$ is the number of the magnetic poles in the rotor, $\Omega_{M}$ is the mechanical rotational speed measured in mechanical rad/s, and $\Omega_{S}$ is the electrical rotational speed measured in electrical $\mathrm{rad} / \mathrm{s}$.

In power systems analysis, it is more convenient to use per unit (p.u.) notation to express the quantities and variables. Basically, a quantity in p.u. is a normalized quantity with respect to an appropriate base value, that is to say, see Kundur (1994):

$$
\text { Quantity in p.u. }=\frac{\text { Quantity }}{\text { Base value of the quantity }}
$$

In order to appreciate the benefit of the p.u. system, it is enough to note that $\omega_{M}$ and $\omega_{S}$ in (1) will be equal in p.u. To elaborate, let the base mechanical rotational speed and the base electrical rotational speed be their rated values, denoted from here on by the superscript $\left({ }^{r}\right)$, i.e. $\Omega_{M}^{r}$, and $\Omega_{S}^{r}$. Then, by using (1), one obtains:

$$
\frac{\Omega_{M}}{\Omega_{M}^{r}}=\frac{\frac{2}{p} \Omega_{S}}{\frac{2}{p} \Omega_{S}^{r}}=\frac{\Omega_{S}}{\Omega_{S}^{r}}=\omega,
$$

where $\omega$ is the synchronous speed in p.u.

Another important common simplification in power systems analysis is the use of the $d q$-frame. Since the magnetic field produced by the armature winding rotates with the same rotational speed of the rotor in steady state, this magnetic field appears stationary from the rotor side. Hence, it can be resolved on two perpendicular axes, that are called the direct $(\mathrm{d})$ axis along the rotor, and quadrature (q) axis perpendicular to the rotor, as shown in Fig. 1. The main advantage of using the $d q$-transformation is to cancel the variations of the mutual and self inductances of the coils due to their dependence on the angle of the rotation. For more details on the $d q$-transformation, the reader is referred to any reference on basic power systems analysis or machines, such as Kundur (1994) and Machowski et al. (2008).

Now, the synchronous generator model, in p.u., and in $d q$-frame, can be described by relations of flux linkages as follows, see Kundur (1994) and Machowski et al. (2008):

$$
\begin{aligned}
\psi_{d} & =-X_{d} i_{d}+X_{F d} i_{F} \\
\psi_{q} & =-X_{q} i_{q} \\
\psi_{F} & =X_{F} i_{F}-X_{F d} i_{d}
\end{aligned}
$$

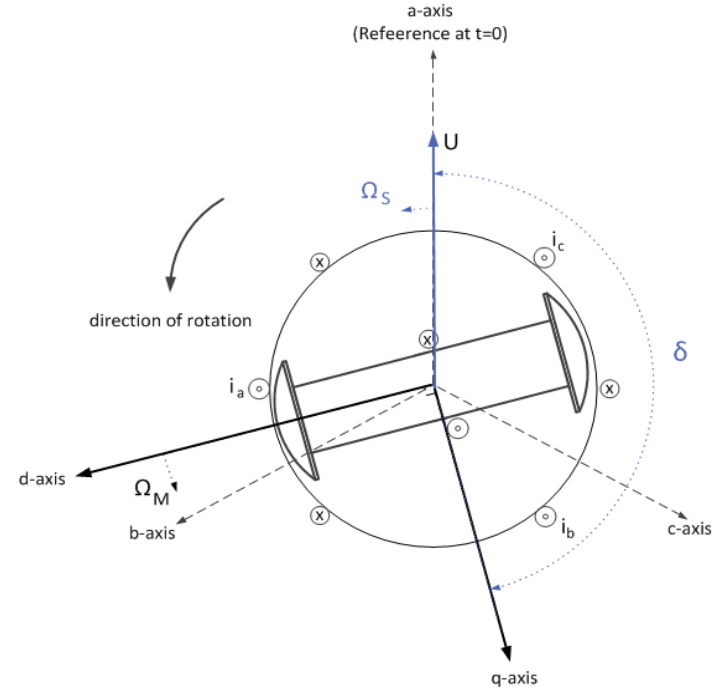

Figure 1: $d q$-frame in the synchronous machine and the angles involved.

and the voltages as follows, see Kundur (1994) and Machowski et al. (2008):

$$
\begin{aligned}
u_{d} & =\dot{\psi}_{d}-\omega \psi_{q}-R_{a} i_{d} \\
u_{q} & =\dot{\psi}_{q}+\omega \psi_{d}-R_{a} i_{q} \\
u_{F} & =\dot{\psi}_{F}+R_{F} i_{F}
\end{aligned}
$$

where $\psi_{d}, \psi_{q}, u_{d}, u_{q}, i_{d}$, and $i_{q}$ are the $d-$, and $q$-axis components of the stator flux linkages, terminal voltage, and stator current, respectively. Further, $\psi_{F}, u_{F}$ and $i_{F}$ are the field circuit flux linkage, voltage, and current respectively. $X_{d}, X_{q}$, an $R_{a}$ are the $d-$, and $q$-axis components of the stator self inductance, and the armature resistance, respectively. It is worth mentioning here that $R_{a}$ is usually much less than the inductances $X_{d}$ and $X_{q}$. Moreover, $X_{F}$, and $R_{F}$ are the field circuit self inductance, and resistance, respectively. $X_{F d}$ is the mutual inductance between the field circuit and stator windings. Recall that all quantities are in p.u.

In the study of power system stability, it is usually acceptable to assume the following:

1. The dynamics of the stator flux linkages $\dot{\psi}_{d}$ and $\dot{\psi}_{q}$ are negleible.

2. $\omega$ in (5a) and (5b) is equal to unity.

For the motivation of the above assumptions the reader is referred to Kundur (1994), Machowski et al. (2008) and the references therein. Hence, the equations in (5) can be rewritten as, see Kundur (1994) and Machowski 
et al. (2008):

$$
\begin{aligned}
u_{d} & =-\psi_{q}-R_{a} i_{d} \\
u_{q} & =\psi_{d}-R_{a} i_{q} \\
u_{F} & =\dot{\psi}_{F}+R_{F} i_{F} .
\end{aligned}
$$

Because the flux linkages are hard to measure in practice, it is also common in the literature on power system stability to use the following transformations, see Kundur (1994) and Machowski et al. (2008):

$$
\begin{aligned}
E_{I} & =X_{F d} i_{F} \\
E_{q}^{\prime} & =\frac{X_{F d}}{X_{F}} \psi_{F} \\
E_{F} & =\frac{X_{F d}}{R_{F}} u_{F},
\end{aligned}
$$

with which the equations in (4) can be rewritten as, see Kundur (1994) and Machowski et al. (2008):

$$
\begin{aligned}
& \psi_{d}=-X_{d} i_{d}+E_{I} \\
& \psi_{q}=-X_{q} i_{q} \\
& E_{q}^{\prime}=E_{I}-\left(X_{d}-X_{d}^{\prime}\right) i_{d}
\end{aligned}
$$

where $X_{d}^{\prime}=X_{d}-\frac{X_{F D}^{2}}{X_{F}}$, and (6c) can be rewritten as:

$$
\dot{E}_{q}^{\prime}=\frac{1}{T_{d 0}^{\prime}}\left(E_{F}-E_{I}\right)
$$

where $T_{d 0}^{\prime}$ is a time constant in seconds. Notice that the only differential equation in this model is the one given in (9), which will be used later in the proposed state-space model. Eq. (9) describes the dynamics of the voltage $E_{q}^{\prime}$, which could be easier to measure than the flux linkage $\psi_{F}$ in (6c). Nevertheless, we would like to replace this with a differential equation that describes the dynamics of the terminal voltage. Thus, inserting (6b) and (8a) in (8c), one can easily obtain the following relation:

$$
E_{q}^{\prime}=u_{q}+X_{d}^{\prime} i_{d}+R_{a} i_{q}
$$

In order to describe the dynamics of the terminal voltage, let us differentiate the above equation, to get:

$$
\dot{E}_{q}^{\prime}=\dot{u}_{q}+X_{d}^{\prime} \frac{d i_{d}}{d t}+R_{a} \frac{d i_{q}}{d t}
$$

Differentiating (6a) and (6b) with respect to time and after neglecting the derivatives of the flux linkages as was done before, the derivatives of the stator currents in terms of the voltages can be described by:

$$
\begin{aligned}
& \frac{d i_{d}}{d t}=-\frac{\dot{u}_{d}}{R_{a}} \\
& \frac{d i_{q}}{d t}=-\frac{\dot{u}_{q}}{R_{a}} .
\end{aligned}
$$

Remark 1 The rate of change of the terminal voltage is much less than the rate of change of the stator currents. This can be seen from (12) because $R_{a}$ is usually small.

However, the voltages in $d q$-frame are expressed, by definition as shown in Fig. 1, as:

$$
\begin{aligned}
& u_{d}=U \sin (\delta) \\
& u_{q}=U \cos (\delta),
\end{aligned}
$$

where $U$ is the terminal voltage in p.u., and $\delta$ is the angular position of the rotor with respect to the rotating reference, as shown in Fig. 1, in electrical rad. Hence, the derivatives of the voltages in $d q$-frame are given by:

$$
\begin{aligned}
& \dot{u}_{d}=\dot{U} \sin (\delta)+\dot{\delta} U \cos (\delta) \\
& \dot{u}_{q}=\dot{U} \cos (\delta)-\dot{\delta} U \sin (\delta) .
\end{aligned}
$$

Finally, inserting (9), (12), and (14) in (11), and by using (8c) and (10), after some algebraic simplifications and by neglecting the term with $R_{a}^{2}$ we obtain:

$$
\begin{aligned}
\dot{U}= & \left(\frac{R_{a}}{T_{d 0}^{\prime} X_{d}^{\prime}}-\dot{\delta}\right) U \cot (\delta)+\frac{R_{a} X_{d}}{T_{d 0}^{\prime} X_{d}^{\prime} \sin (\delta)} i_{d} \\
& -\frac{R_{a}}{T_{d 0}^{\prime} X_{d}^{\prime} \sin (\delta)} E_{F} .
\end{aligned}
$$

The rotor is supposed to rotate with the synchronous speed, and thus the angle $\delta$ is supposed to be constant in steady state. Nevertheless, in the general form, the angle $\delta$ will change as, see Kundur (1994) and Machowski et al. (2008):

$$
\delta=\Omega_{S} t-\Omega_{S}^{r} t+\delta_{0},
$$

where $\delta_{0}$ is the initial value. Then, the dynamics of the rotor angle $\delta$ can be obtained, by differentiating the above equation, and by using (3), to be:

$$
\dot{\delta}=\Omega_{S}^{r}(\omega-1) .
$$

Finally, we need to calculate the electromagnetic torque of the synchronous machine. As mentioned earlier, the rotating magnetic field from the armature windings tries to catch the magnetic field of the rotor, and thus a torque is created. If the machine is working as a motor, the current will be fed into the stator, the torque developed will be mechanical and thus the rotor keeps moving. If the machine is working as a generator, the rotor is moved by an external prime mover, and an electromagnetic torque is produced to induce currents in the armature windings. The electromagnetic torque $q_{S}$, in p.u., can be expressed by:

$$
q_{S}=\psi_{d} i_{q}-\psi_{q} i_{d}
$$


which can be simplified by using (4) and (6), and by neglecting $R_{a}$, to:

$$
\begin{aligned}
q_{S} & =u_{q} \frac{u_{d}}{X_{q}}+u_{d} i_{d}=\frac{1}{X_{q}} U^{2} \sin (\delta) \cos (\delta)+U i_{d} \sin (\delta) \\
& =\frac{1}{2 X_{q}} U^{2} \sin (2 \delta)+U i_{d} \sin (\delta) .
\end{aligned}
$$

\subsection{Diesel Engine}

Diesel engines belong to the class of the compression ignition (CI) engines, that do not need a spark to start the ignition, of internal combustion (IC) engines. Diesel engines are the most efficient IC engines, they can achieve over 50\% efficiency according to Guzzella and Amstutz (1998). Diesel engines suffer from two main problems: low power density and nitric oxygen $\left(\mathrm{NO}_{\mathrm{x}}\right)$ emission, Guzzella and Onder (2010). The problem with power density is solved by using a turbocharger, while the problem with the $\mathrm{NO}_{\mathrm{x}}$ emission is overcome by using exhaust gas recircluation (EGR). Basically, a turbocharger is a compressor that pushes more air into the cylinder and thus more fuel is burnt in the same volume. The EGR, on the other hand, is a technique that depends on valves that allow the exhaust gas to be fed back into the cylinder, and thus reducing the $\mathrm{NO}_{\mathrm{x}}$ emissions. To get more details on how the EGR does that, the reader is referred to Heywood (1988), Guzzella and Amstutz (1998), and Guzzella and Onder (2010) and the references therein.

Actually, discussing the control of the Diesel engine in details is beyond the scope of this work. However, we need to emphasize that the modern Diesel engine is a complicated system by itself that contains several interconnected control loops, mainly the air path, fuel path, and exhaust gas path, that need to be coordinated. For example, increasing the EGR valve opening reduces the $\mathrm{NO}_{\mathrm{x}}$, but at the same time it increases the brake specific fuel consumption (bsfc) and the particulates emission. Hence, the best performance of the Diesel engine is a trade-off among many factors. That is why, it is very important when designing a controller to choose the target taking in consideration the other control loops.

In order to design a controller that regulates the shaft speed and the terminal voltage in a Genset, one is interested in, mainly, the torque provided by the Diesel engine. Many models have been suggested in the literature for the torque produced by the Diesel engine depending on the objective of the proposed model. For the control of a Genset, it is enough to develop a simplified model based on the speed governor. Fossen (1994) stated three models described by transfer functions between the output, that is the torque produced by the Diesel engine $Q_{E}$ in $\mathrm{Nm}$, and the input that is $y$ the fuel pump index. The most common of them is the one which we re-write here in time domain, see Fossen (1994):

$$
\dot{Q}_{E}=\frac{-1}{T_{y}} Q_{E}+\frac{K_{y}}{T_{y}} y(t-\tau),
$$

where $T_{y}$ is a time constant, $K_{y}$ is a gain constant, and $\tau$ is a time delay or dead time, in seconds, of the Diesel engine given by, see Fossen (1994):

$$
\tau=\frac{1}{2 N_{c} n_{E}},
$$

where $N_{c}$ is the number of the cylinders in the Diesel engine, and $n_{E}$ is its speed in revolutions per second (rps). By using this model, we can consider the engine torque as another state in the proposed model. This stipulates that the torque is available for measuring. In fact, torque is not easy to measure in practice, hence observers are required to overcome this problem. Alternatively, one can simplify the model above by neglecting the engine torque dynamics $\dot{Q}_{E}$. This simplification is very common in literature and practice, see e.g. Hansen (2000), and it implies that the torque is considered equal to the input control multiplied by its gain $K_{y} y(t-\tau)$. Anyway, by using the speed governor, the amount of fuel is adjusted by controlling the rack position based on the error signal of the speed, which can be measured easily. Now, if we would like to combine the action of the speed governor and the AVR, a state-space model is needed because of the nonlinearity of the system. Although the model (20) was used by Hansen et al. (2001) to propose a controller for marine Genset, we believe that this model may not be sufficient for the following two reasons. First, the air dynamics are not taken in consideration. Then, the engine torque is difficult to measure.

Many researchers have been trying to propose a model to determine the torque in order to avoid measuring it. The model we adopt in this work is the one suggested in Jensen et al. (1991). The torque produced by the Diesel engine $Q_{E}$ is the difference between the indicated torque $Q_{\text {ind }}$ and the frictional torque $Q_{\mathrm{Fr}}$, see Heywood (1988). The indicated torque can be described by the following well-known relation, see Kao and Moskwa (1994) and Guzzella and Amstutz (1998):

$$
Q_{\text {ind }}=m_{\phi} H_{l} \eta_{\text {ind }},
$$

where $H_{l}$ is the fuel lower heating value in $\mathrm{J} / \mathrm{kg}$ which is constant for Diesel fuel and it will be considered in all simulations equal to $42 \mathrm{MJ} / \mathrm{kg}$. Furthermore, $m_{\phi}$ is the fuel mass injected in $\mathrm{kg}$, and $\eta_{\text {ind }}$ denotes the indicated efficiency. Jensen et al. (1991) proposed a mean-value model of the different parts and loops of a small turbocharged Diesel engine used in vehicular systems. They analysed experimental data to show that 
the indicated efficiency is a function of the engine speed and the fuel/air equivalence ratio $\Phi$ as, see Jensen et al. (1991):

$$
\eta_{\text {ind }}=\left(a_{1}+a_{2} \Omega_{E}+a_{3} \Omega_{E}^{2}\right)\left(1-a_{4} \Phi^{a_{5}}\right),
$$

where $\Omega_{E}$ is the rotational speed of the engine in mechanical rad $/ \mathrm{s}$, and $a_{1}, \ldots, a_{5}$ are parameters of appropriate dimensions. According to the definitions in Heywood (1988), the fuel/air equivalence ratio is the reciprocal of the relative air/fuel ratio $\lambda$ defined by, see Heywood (1988):

$$
\Phi^{-1}=\lambda=\frac{A / F}{(A / F)_{s}},
$$

where the subscript $\left({ }_{s}\right)$ denotes the value corresponding to the stoichiometric combustion (see Subsection 4.2), and $A / F$ is the air/fuel ratio given by, see Heywood (1988):

$$
A / F=\frac{\dot{m}_{a}}{\dot{m}_{f}}
$$

with $\dot{m}_{a}$ and $\dot{m}_{f}$ represent the flow rate of the air and the fuel, respectively. On the other hand, the frictional torque $Q_{\mathrm{Fr}}$ is described by, see Kao and Moskwa (1994) and Heywood (1988):

$$
Q_{\mathrm{Fr}}=\frac{p_{\mathrm{fme}} V_{d}}{2 \pi \nu},
$$

where $V_{d}$ denotes the displacement volume in $\mathrm{m}^{3}, \nu$ is the number of revolutions for each power stroke per cycle, and $p_{\text {fme }}$ is the friction mean effective pressure in $\mathrm{Pa}$. Heywood (1988) deduced from motoring tests of different types of Diesel engines that friction mean effective pressure $p_{\text {fme }}$ can be described by:

$$
p_{\text {fme }}=1000\left(C_{1}+0.048 \frac{60}{2 \pi} \Omega_{E}+0.4 \bar{S}_{p}^{2}\right),
$$

where $C_{1}$ is a constant in $\mathrm{kPa}$ that depends on the engine type, $\bar{S}_{p}$ is the mean piston speed in $\mathrm{m} / \mathrm{s}$. The last thing we need to take in consideration is the time delay given in (21), because the indicated torque $Q_{\text {ind }}$ requires some time to have effect. Thus, the final model of the torque produced by the engine is given by:

$$
\begin{aligned}
Q_{E}(t)= & Q_{\text {ind }}(t-\tau)-Q_{\mathrm{Fr}}(t) \\
= & H_{l}\left(a_{1}+a_{2} \Omega_{E_{\tau}}+a_{3} \Omega_{E_{\tau}}^{2}\right)\left(1-a_{4} \Phi_{\tau}^{a_{5}}\right) m_{\phi_{\tau}} \\
& -\frac{1000 V_{d}}{2 \pi \nu}\left(C_{1}+0.048 \frac{60}{2 \pi} \Omega_{E}+0.4 \bar{S}_{p}^{2}\right),
\end{aligned}
$$

where the subscript ${ }_{\tau}$ ) denotes the delayed signal, e.g. $\Omega_{E_{\tau}}=\Omega_{E}(t-\tau)$.

Actually, Jensen et al. (1991) claimed that the model above can be used for large Diesel engines, as well. However, analysing experimental data to verify this claim is beyond the scope of this work, so we depend on the previous claim to design the proposed controller.

\subsection{Shaft Dynamics}

One of the most fundamental relations in the study of power system stability is the so-called swing equation. This equation connects the mechanical and electrical aspects of the generators. The synchronous machine and the prime mover are connected mechanically through a shaft and sometimes a gear box. Thus, the rotational speed of the engine is as same as that of the shaft, while the speed of the synchronous machine is related to that of the engine by:

$$
\Omega_{M}=R_{M} \Omega_{E},
$$

where $R_{M}$ is the gear ratio, and $\Omega_{M}$ is the mechanical rotational speed of the synchronous machine in mechanical rad/s, as defined in (1). Then, applying Newton's second law of rotating objects to the mechanical system, one gets:

$$
\left(I_{E}+R_{M}^{2} I_{M}\right) \dot{\Omega}_{E}=Q_{E}-R_{M} Q_{S}-R_{M} Q_{D},
$$

where $Q_{E}$ is as before, $Q_{S}$ denotes the electromagnetic torque consumed by the synchronous machine in $\mathrm{Nm}$, and $Q_{D}$ is a damping torque. Further, $I_{E}$ and $I_{M}$ are the moment of inertia, in $\mathrm{kg} \mathrm{m}^{2}$, of the engine and synchronous machine, respectively.

Remark 2 Note that, in the model in eq.(30), the moment of inertia of the shaft is neglected, this should not be a problem since this quantity is constant and it can be added to the moment of inertia of the engine or the machine. Further, the shaft introduces some kind of friction to the motion. One can find many models to include the opposing torque resulting from this friction, see e.g. Pivano et al. (2007) and the references therein. The simplest one may be to assume the frictional torque proportional to the rotational speed. In this work, the frictional torque is neglected because including it should not affect the proposed strategy to design the controller, as long as the frictional torque function is "nice", and hence can be added to the load torque.

Following the procedure in Kundur (1994), define the p.u. inertia constant $H_{T}$ as the ratio of the stored kinetic energy at rated speed to the base value of the apparent power of the synchronous machine $S_{\text {base }}$ in VA, i.e.:

$$
H_{T}=\frac{1}{2} \frac{\left(I_{E}+R_{M}^{2} I_{M}\right)\left(\Omega_{E}^{r}\right)^{2}}{S_{\text {base }}} .
$$

Then, substitute $H_{T}$ in (30) to get:

$$
2 H_{T} \frac{S_{\text {base }}}{\left(\Omega_{E}^{r}\right)^{2}} \dot{\Omega}_{E}=Q_{E}-R_{M} Q_{S}-R_{M} Q_{D},
$$

which can be simplified to:

$$
2 H_{T} \frac{d}{d t}\left(\frac{\Omega_{E}}{\Omega_{E}^{r}}\right)=\frac{Q_{E}}{S_{\text {base }} / \Omega_{E}^{r}}-\frac{R_{M} Q_{S}}{S_{\text {base }} / \Omega_{E}^{r}}-\frac{R_{M} Q_{D}}{S_{\text {base }} / \Omega_{E}^{r}} .
$$


Now, choose the base quantities as follows:

$$
\begin{aligned}
\Omega_{E_{\text {base }}} & =\Omega_{E}^{r} \\
Q_{E_{\text {base }}} & =S_{\text {base }} / \Omega_{E}^{r} \\
Q_{S_{\text {base }}} & =S_{\text {base }} / \Omega_{M}^{r}=S_{\text {base }} / R_{M} \Omega_{E}^{r} \\
Q_{D_{\text {base }}} & =S_{\text {base }} / \Omega_{M}^{r}=S_{\text {base }} / R_{M} \Omega_{E}^{r} .
\end{aligned}
$$

Note that the p.u. speed of the engine is equal to the p.u. speed of the machine because:

$$
\omega=\frac{\Omega_{M}}{\Omega_{M}^{r}}=\frac{\Omega_{M} / R_{M}}{\Omega_{M}^{r} / R_{M}}=\frac{\Omega_{E}}{\Omega_{E}^{r}},
$$

from (3) and (29). Now, substitute the base quantities in (33) to obtain:

$$
\dot{\omega}=\frac{1}{2 H_{T}}\left(q_{E}-q_{S}-q_{D}\right),
$$

where $q_{S}$ is the electromagnetic torque in p.u. given by (19), $q_{D}$ is the damping torque proportional to speed deviation as, Kundur (1994):

$$
q_{D}=k_{D}(\omega-1),
$$

with $k_{D}$ is the damping coefficient, and $q_{E}$ is the torque produced by the engine in p.u. notation, given by $\frac{Q_{E}}{S_{\text {base }} / \Omega_{E}^{r}}$.

\section{Simplified Model and Control Design}

To begin with, let us define some parameters to simplify the notation.

$$
\begin{aligned}
k_{E} & =\frac{H_{l} \Omega_{E}^{r}}{S_{\text {base }}} \\
\Theta_{E} & =1-a_{4} \Phi^{a_{5}} \\
\tilde{a}_{2} & =a_{2} \Omega_{E}^{r} \\
\tilde{a}_{3} & =a_{3}\left(\Omega_{E}^{r}\right)^{2} \\
k_{f_{1}} & =k_{D}+48 \frac{60}{2 \pi} \frac{V_{d}\left(\Omega_{E}^{r}\right)^{2}}{2 \pi \nu S_{\text {base }}} \\
k_{f_{2}} & =\frac{1000 V_{d} \Omega_{E}^{r}}{2 \pi \nu S_{\text {base }}}\left(C_{1}+0.4 \bar{S}_{p}^{2}\right)-k_{D}
\end{aligned}
$$

Because the model we have so far is highly non-linear, we need to make the following assumptions to design the controller:

1. The time delay $\tau$ is negligible.

2. The quantity $\Theta_{E}$ is constant.

The assumptions above, especially the second one, may seem restrictive. However, we show in the next section that without these assumptions the proposed controller still performs satisfactorily.

Now, the proposed model that can be used for control design of the shaft speed and the terminal voltage of the Genset, is described by the following three-state model:

$$
\begin{aligned}
\dot{\delta}= & \Omega_{S}^{r}(\omega-1) \\
\dot{\omega}= & \frac{1}{2 H_{T}}\left(k_{E} \Theta_{E}\left(a_{1}+\tilde{a}_{2} \omega+\tilde{a}_{3} \omega^{2}\right) m_{\phi}-\frac{1}{2 X_{q}} U^{2} \sin (2 \delta)\right. \\
& \left.-U i_{d} \sin (\delta)-k_{f_{1}} \omega-k_{f_{2}}\right) \\
\dot{U}= & \left(\frac{R_{a}}{T_{d 0}^{\prime} X_{d}^{\prime}}-\Omega_{S}^{r}(\omega-1)\right) U \cot (\delta)+\frac{R_{a} X_{d}}{T_{d 0}^{\prime} X_{d}^{\prime} \sin (\delta)} i_{d} \\
& -\frac{R_{a}}{T_{d 0}^{\prime} X_{d}^{\prime} \sin (\delta)} E_{F} .
\end{aligned}
$$

Note that the states of this model are the rotor angle, the shaft speed and the terminal voltage, which are easily measurable. The manipulated input controls are the fuel mass injected in the engine $m_{\phi}$, and the voltage proportional to the field circuit voltage $E_{F}$. Finally, we model the stator current in $d$-axis $i_{d}$ as a disturbance that is available for measurement.

Let us denote the states in the model above by $\mathbf{x}$ given by:

$$
\mathbf{x}=\left[x_{1}, x_{2}, x_{3}\right]^{T}=[\delta, \omega, U]^{T} .
$$

Let also the input controls of the above systems be $\mathbf{u}=\left[u_{1}, u_{2}\right]^{T}=\left[m_{\phi}, E_{F}\right]^{T}$. Let further the current $i_{d}$ be denoted by $d$. Then, the final model writes:

$$
\dot{\mathbf{x}}=f(\mathbf{x})+g_{1}(\mathbf{x}) u_{1}+g_{2}(\mathbf{x}) u_{2}+p(\mathbf{x}) d,
$$

where,

$$
\begin{gathered}
f(\mathbf{x})=\left[\begin{array}{c}
\Omega_{S}^{r}\left(x_{2}-1\right) \\
\left.\frac{-1}{2 H_{T}}\left(\frac{1}{2 X_{q}} x_{3}^{2} \sin \left(2 x_{1}\right)+k_{f_{1}} x_{2}+k_{f_{2}}\right)\right) \\
\left(\frac{R_{a}}{T_{d 0}^{\prime} X_{d}^{\prime}}-\Omega_{S}^{r}\left(x_{2}-1\right)\right) x_{3} \cot \left(x_{1}\right)
\end{array}\right], \\
g_{1}(\mathbf{x})=\left[\begin{array}{c}
0 \\
\frac{k_{E} \Theta_{E}}{2 H_{T}}\left(a_{1}+\tilde{a}_{2} x_{2}+\tilde{a}_{3} x_{2}^{2}\right) \\
0
\end{array}\right], \\
g_{2}(\mathbf{x})=\left[\begin{array}{c}
0 \\
0 \\
-\frac{R_{a}}{T_{d 0}^{\prime} X_{d}^{\prime} \sin \left(x_{1}\right)}
\end{array}\right],
\end{gathered}
$$

and

$$
p(\mathbf{x})=\left[\begin{array}{c}
0 \\
\frac{-1}{2 H_{T}} x_{3} \sin \left(x_{1}\right) \\
\frac{R_{a} X_{d}}{T_{d 0}^{\prime} X_{d}^{\prime} \sin \left(x_{1}\right)}
\end{array}\right] .
$$

To this end, we use feedback linearisation to design a controller for this system. It is important in feedback 
linearisation to choose the appropriate outputs. As mentioned before, the sought controller is supposed to regulate the terminal voltage and the shaft speed, simultaneously. Thus, the terminal voltage is definitely one of the outputs. Instead of the shaft speed as a second output, we choose the angle for two reasons. Firstly, the model will be exactly feedback linearisable with these outputs. Secondly, controlling the rotor angle ensures the stability of the speed and thus the frequency of the generated currents. Hence, let the outputs be:

$$
\begin{aligned}
& y_{1}=h_{1}(\mathbf{x})=x_{1}-x_{1}^{d} \\
& y_{2}=h_{2}(\mathbf{x})=x_{3}-x_{3}^{d},
\end{aligned}
$$

where $x_{1}^{d}$ and $x_{3}^{d}$ are the desired angle and output voltage to be tracked. Following the well-known procedure for feedback linearisation as in Isidori (1989) e.g., define the external state $z_{1}=h_{1}(\mathbf{x})=x_{1}-x_{1}^{d}$, the derivative is obtained to be:

$$
\dot{z}_{1}=L_{f} h_{1}(\mathbf{x})=\Omega_{S}^{r}\left(x_{2}-1\right),
$$

where $L_{f} h_{1}(\mathbf{x})$ denotes the Lie derivative of $h_{1}(\mathbf{x})$ along the vector field $f(\mathbf{x})$. Then, define the state $z_{2}=\Omega_{S}^{r}\left(x_{2}-1\right)$ whose derivative is given by:

$$
\begin{aligned}
\dot{z}_{2}= & L_{f}^{2} h_{1}(\mathbf{x})+u_{1} L_{g_{1}} L_{f} h_{1}(\mathbf{x})+u_{2} L_{g_{2}} L_{f} h_{1}(\mathbf{x}) \\
& +d L_{p} L_{f} h_{1}(\mathbf{x}),
\end{aligned}
$$

in which $L_{g_{2}} L_{f} h_{1}(\mathbf{x})=0$. Thus, the model in eq.(40) has a relative degree two with respect to output $y_{1}$, i.e., $r_{1}=2$. Moreover, define the state $z_{3}=h_{2}(\mathbf{x})=$ $x_{3}-x_{3}^{d}$, and differentiate to get:

$\dot{z}_{3}=L_{f} h_{2}(\mathbf{x})+u_{1} L_{g_{1}} h_{2}(\mathbf{x})+u_{2} L_{g_{2}} h_{2}(\mathbf{x})+d L_{p} h_{2}(\mathbf{x})$,

where $L_{g_{1}} h_{2}(\mathbf{x})=0$. Hence, the relative degree with respect to the output $y_{2}$ is one. Since, $r_{1}+r_{2}=3$, the model can be feedback linearised exactly, and hence no zero dynamics will be encountered. Choosing the input control laws as follows:

$$
\begin{aligned}
& u_{1}=\frac{1}{L_{g_{1}} L_{f} h_{1}(\mathbf{x})}\left(-L_{f}^{2} h_{1}(\mathbf{x})-d L_{p} L_{f} h_{1}(\mathbf{x})+v_{1}\right) \\
& u_{2}=\frac{1}{L_{g_{2}} h_{2}(\mathbf{x})}\left(-L_{f} h_{2}(\mathbf{x})-d L_{p} h_{2}(\mathbf{x})+v_{2}\right)
\end{aligned}
$$

where the explicit Lie derivatives are given by:

$$
\begin{aligned}
L_{f}^{2} h_{1}(\mathbf{x}) & =\frac{-\Omega_{S}^{r}}{2 H_{T}}\left(\frac{1}{2 X_{q}} x_{3}^{2} \sin \left(2 x_{1}\right)+k_{f_{1}} x_{2}+k_{f_{2}}\right) \\
L_{g_{1}} L_{f} h_{1}(\mathbf{x}) & =\frac{\Omega_{S}^{r} k_{E} \Theta_{E}}{2 H_{T}}\left(a_{1}+\tilde{a}_{2} x_{2}+\tilde{a}_{3} x_{2}^{2}\right) \\
L_{p} L_{f} h_{1}(\mathbf{x}) & =\frac{-\Omega_{S}^{r}}{2 H_{T}} x_{3} \sin \left(x_{1}\right),
\end{aligned}
$$

and

$$
\begin{aligned}
L_{f} h_{2}(\mathbf{x}) & =\left(\frac{R_{a}}{T_{d 0}^{\prime} X_{d}^{\prime}}-\Omega_{S}^{r}\left(x_{2}-1\right)\right) U \cot \left(x_{1}\right) \\
L_{g_{2}} h_{2}(\mathbf{x}) & =-\frac{R_{a}}{T_{d 0}^{\prime} X_{d}^{\prime} \sin \left(x_{1}\right)} \\
L_{p} h_{2}(\mathbf{x}) & =\frac{R_{a} X_{d}}{T_{d 0}^{\prime} X_{d}^{\prime} \sin \left(x_{1}\right)},
\end{aligned}
$$

the external dynamics will be:

$$
\dot{\mathbf{z}}=\left[\begin{array}{c}
\dot{z}_{1} \\
\dot{z}_{2} \\
\dot{z}_{3}
\end{array}\right]=A_{z} \mathbf{z}+B_{z} \mathbf{v}
$$

where $\mathbf{v}=\left[v_{1}, v_{2}\right]^{T}$ is an auxiliary stabilizing input control vector, and

$$
A_{z}=\left[\begin{array}{lll}
0 & 1 & 0 \\
0 & 0 & 0 \\
0 & 0 & 0
\end{array}\right], B_{z}=\left[\begin{array}{ll}
0 & 0 \\
1 & 0 \\
0 & 1
\end{array}\right] \text {. }
$$

The model above is linear and controllable, hence one can find a control law:

$$
\mathbf{v}=-\mathbf{K}_{\mathbf{p}} \mathbf{z}
$$

that stabilizes the system in (48) by pole placement, for example. Note that the disturbance is used as a feed forward signal in this control law and hence completely decoupled from the output.

\subsection{Simulation Results}

The model in eq.eq.(40) with the control input laws in (45) was simulated in MATLAB R2012b. The synchronous machine we used for our simulations has the parameters listed in Table 1. Finding the coefficients $a_{1}, \ldots, a_{5}$ of the indicated efficiency model given in (23) is challenging. The specification sheets of Diesel engines do not include such functions. Hence, in order to find those parameters one needs to get experimental data and perform multi-variable non-linear regression or curve-fitting techniques on them. However, that is outside the scope of this work. Since we are interested in assessing the proposed controller, and we assumed the validity of the claim proposed in Jensen et al. (1991) that the indicated efficiency can be approximated by a function of the form in (23), reasonable values are chosen in this work. Table 2 lists representative parameters of a Diesel engine. Further, $\Theta_{E}$ in (38) was assumed constant of 0.4 , as stated before. Finally, the desired outputs to be tracked in (41) were set to 1 .

It may not be suitable to simulate the model from zero initial states and see how to it will reach the desired 
values because Diesel engines are usually started for some time and then it will be loaded gradually. Thus, we chose to run the simulations from steady-state values, namely, $\delta(0)=1, \omega(0)=1$, and $U(0)=1$, and then a perturbation was applied to see how the proposed controller would perform. The stator current in $d$-axis $i_{d}$ was assumed of sinusoidal nature over $20 \mathrm{~s}$, the simulation horizon, as:

$$
i_{d}=1+0.04 \sin (5 \pi t)
$$

and it was fed forward to the controller. The initial values of the states were assumed equal to the steady state value. Then, a sudden step of 0.1 p.u. in the load torque, or in the terminal voltage, was introduced from $t=5 \mathrm{~s}$ till $t=7 \mathrm{~s}$, as a perturbation that is not seen by the controller. The gain matrix $\mathbf{K}_{\mathbf{p}}$ in (50) was obtained by pole placement.

In order to justify the choice of the poles, we need to notice from the structure of the matrices of the linearised model in (49), that the gain $\mathbf{K}_{\mathbf{p}}$ for any poles vector $\left[\begin{array}{lll}p_{1} & p_{2} & p_{3}\end{array}\right]$ has the following form:

$$
\mathbf{K}_{\mathbf{p}}=\left[\begin{array}{ccr}
p_{2} p_{3} & -\left(p_{2}+p_{3}\right) & 0 \\
0 & 0 & -p_{1}
\end{array}\right]
$$

Thus, the first pole controls the output voltage state $z_{3}=x_{3}-x_{3}^{d}=U-1$, while the last 2 poles control the angle and the speed. In fact, choosing the gains of the angle and speed states is a little tricky. To elucidate, high gains make the controller aggressive, which leads to an increase in the first control input signal, that is the fuel mass input $u_{1}=m_{\phi}$. On the other hand, low gains make the angle drop to lower values, and hence the second control input $u_{2}$, that is the field circuit input voltage $E_{F}$, may reach undesired values or even explode due to the sine function in the denominator of the control law in (45), as can be seen in the Lie derivatives. Thus, choosing the poles and hence the gains is a trade-off between reducing the fuel mass input $m_{\phi}$ and avoiding unreasonable levels of the field circuit voltage input $E_{F}$. In order to illustrate the aforementioned analysis, two gains were tried in the simulations. Gain $\mathbf{K}_{\mathbf{1}}$ obtained by placing the poles at [-5 -20 -5], and gain $\mathbf{K}_{\mathbf{2}}$ obtained by placing the poles at [-5 $\left.-20-25\right]$. As mentioned earlier, a sudden increase step of 0.1 p.u. in the load torque was introduced between $t=5 \mathrm{~s}$ and $t=7 \mathrm{~s}$. The results are shown in Fig. 2.

At $t=5 \mathrm{~s}$ when a sudden load increase is introduced, as can be seen from Fig. 2, the speed decreases abruptly, and thus the angle will drop to another steady state-value. Note that the new steadystate value of the angle is not equal to the desired one, but it will stay stable because the speed reaches stability quickly. On the other hand, at $t=7 \mathrm{~s}$, this increased sudden load is removed making the speed increase, and hence the angle, until the angle reaches the desired steady-state value and the speed stabilises again.

The terminal voltage is not affected by this sudden increase, however. This may not be realistic, since one expects a disruption in the terminal voltage when the rotational speed is disrupted. The reason behind that is the fact that the proposed model is too simplified that it is best suited for control design, and it may not be suitable for accurate simulations. Anyway, since we are interested in the behaviour of the controllers and the interaction between them to stabilize the states, this model probably suffices for the mentioned targets. Although the terminal voltage $U$ is not affected by this sudden increase in the load torque, the field circuit voltage $E_{F}$ increases to compensate for the decrease in the speed at $t=5 \mathrm{~s}$. Conversely, $E_{F}$ decreases at $t=7 \mathrm{~s}$ to compensate for the increase in the speed.

Now let us try to comment on the choice of the gains. By using the function place in MATLAB, the gains $\mathbf{K}_{\mathbf{1}}$ and $\mathbf{K}_{\mathbf{2}}$ were obtained at the given poles to be:

$$
\begin{aligned}
\text { at poles } & {\left[\begin{array}{lll}
-5 & -20-5
\end{array}\right] \longrightarrow \mathbf{K}_{\mathbf{1}}=\left[\begin{array}{ccc}
100 & 25 & 0 \\
0 & 0 & 5
\end{array}\right] } \\
\text { at poles } & {[-5-20-25] \longrightarrow \mathbf{K}_{\mathbf{2}}=\left[\begin{array}{ccc}
500 & 45 & 0 \\
0 & 0 & 5
\end{array}\right] }
\end{aligned}
$$

So, the elements of $\mathbf{K}_{\mathbf{1}}$ corresponding to the angle and speed are less than those of $\mathbf{K}_{\mathbf{2}}$. As can be seen in Fig. 2, when the load torque step is introduced at $t=5$ $\mathbf{s}$, the angle drops to a lower value with $\mathbf{K}_{\mathbf{1}}$ because the speed takes longer time to retain its desired value, 1 p.u. Thus, the field circuit input voltage $E_{F}$ increases to higher values. In opposition, increasing the gains of the angle and speed makes the speed retain its desired values quickly by increasing the first input control $\left(m_{\phi}\right)$. In a nutshell, reducing the gains of the angle and speed moves the burden of retaining the steady-state values from the first input control $\left(m_{\phi}\right)$ to the second input control $\left(E_{F}\right)$. Hence, fine tuning is required to choose the gains because it is a trade-off between keeping the mass fuel input minimized and keeping the field circuit input at reasonable level. However, the most important advantage of this controller is the interconnection between the two input controls. That is to say, both inputs contribute to keeping the system stable at the same time and not separately.

Let us now see how the controllers would react if the terminal current is perturbed. A sudden increase of 0.25 p.u. in the terminal current in the $d$-axis $i_{d}$ was introduced between $t=5 \mathrm{~s}$ and $t=7 \mathrm{~s}$ without feeding it forward to the controller. The results obtained are depicted in Fig. 3. It is shown in the figure that 
Table 1: The Parameters of the synchronous machine

\begin{tabular}{|c||c|c|c|c|c|c|}
\hline Quantity & $\begin{array}{c}I_{M} \\
\mathrm{~kg} \mathrm{~m}^{2}\end{array}$ & $\begin{array}{c}X_{d} \\
\text { p.u. }\end{array}$ & $\begin{array}{c}X_{d}^{\prime} \\
\text { p.u. }\end{array}$ & $\begin{array}{c}X_{q} \\
\text { p.u. }\end{array}$ & $\begin{array}{c}R_{a} \\
\text { p.u. }\end{array}$ & $\begin{array}{c}\text { Frequency } \\
\mathrm{Hz}\end{array}$ \\
\hline Value & 250 & 2.0 & 0.25 & 1.0 & 0.004 & 60 \\
\hline \hline Quantity & $k_{D}$ & $T_{d 0}^{\prime}$ & $\begin{array}{c}S_{\text {base }} \\
\text { p.u. }\end{array}$ & $\begin{array}{c}\Omega_{S}^{r} \\
\text { MVA }\end{array}$ & $p$ & $\begin{array}{c}U_{\text {base }} \\
\text { Mad } / \mathrm{s}\end{array}$ \\
\hline Value & 0.05 & 3.0 & 3.0 & 377 & 6 & 360 \\
\hline
\end{tabular}

Table 2: The Parameters of the Diesel engine

\begin{tabular}{|c||c|c|c|c|c|c|}
\hline Quantity & $\begin{array}{c}I_{E} \\
\mathrm{~kg} \mathrm{~m}^{2}\end{array}$ & $\begin{array}{c}V_{d} \\
\mathrm{dm}^{3}\end{array}$ & $\begin{array}{c}C_{1} \\
\mathrm{kPa}\end{array}$ & $N_{c}$ & $\begin{array}{c}\Omega_{E}^{r} \\
\mathrm{rad} / \mathrm{s}\end{array}$ & $\begin{array}{c}\text { Rated Power } \\
\mathrm{MW}\end{array}$ \\
\hline Value & 1800 & 193 & 110 & 6 & 78.54 & 3 \\
\hline \hline Quantity & $(A / F)_{s}$ & $R_{M}$ & $\begin{array}{c}\bar{S}_{p} \\
\mathrm{~m} / \mathrm{s}\end{array}$ & $a_{1}$ & $\begin{array}{c}a_{2} \\
\mathrm{~s} / \mathrm{m}\end{array}$ & $\begin{array}{c}a_{3} \\
\mathrm{~s}^{2} / \mathrm{m}^{2}\end{array}$ \\
\hline Value & 14.9 & 1.6 & 10 & 0.674 & $2.41 \times 10^{-4}$ & $-3.28 \times 10^{-8}$ \\
\hline
\end{tabular}

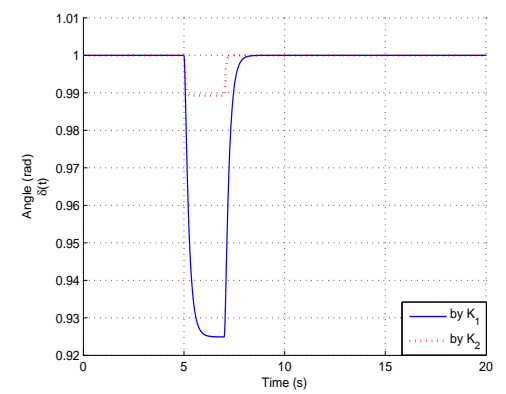

(a) Angle $x_{1}(t)=\delta$

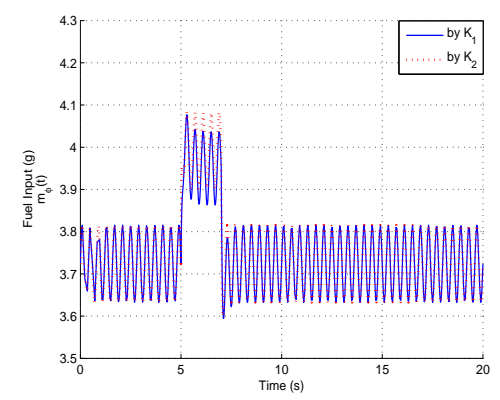

(d) Fuel mass input $u_{1}(t)=m_{\phi}$

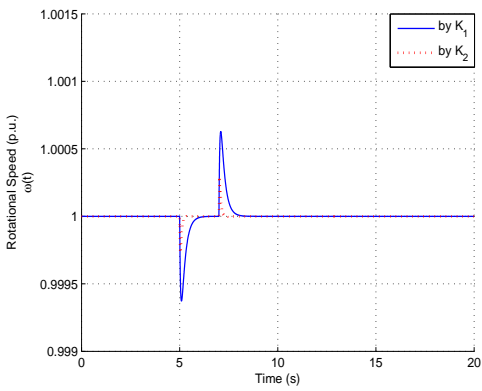

(b) Rotational speed $x_{2}(t)=\omega$

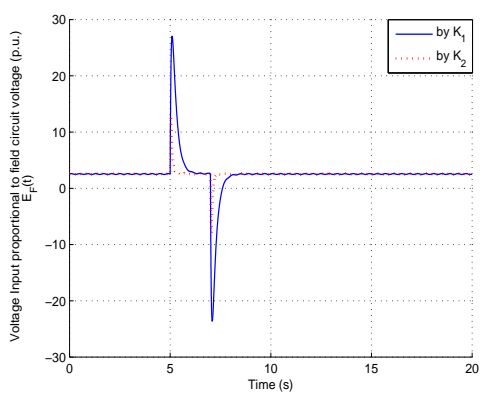

(e) Field circuit input $u_{2}(t)=E_{F}$

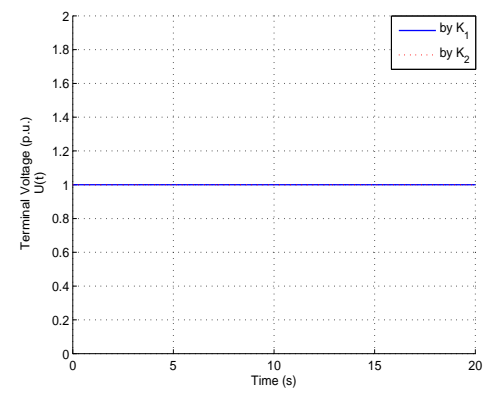

(c) Terminal Voltage $x_{3}(t)=U$

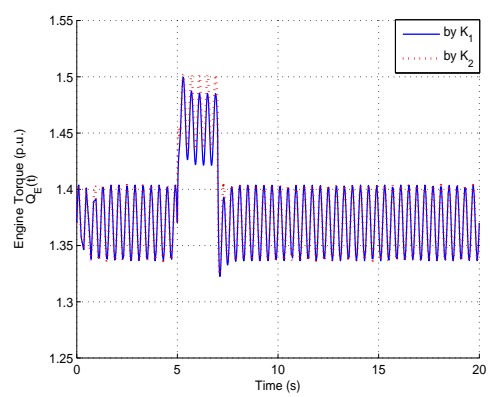

(f) Engine Torque $Q_{E}(t)$

Figure 2: Results of the simulation of the model in eq.(40) with a sudden step in the load torque by the gains: $\mathbf{K}_{\mathbf{1}}$ (solid) and $\mathbf{K}_{\mathbf{2}}$ (dotted)

all states are affected by this step because the current affects the electromagnetic torque and the terminal voltage. The gains $\mathbf{K}_{\mathbf{1}}$ and $\mathbf{K}_{\mathbf{2}}$ have almost the same controlling effect. Increasing the gain increases the fuel mass input $m_{\phi}$, and reduces the field circuit voltage $E_{F}$. One more time, we see that the two input controls are reacting with each other to keep stability.

\section{Uncertainties}

In this section, we discuss the performance of the proposed controller when the time delay and air/fuel ratio are taken in consideration. 


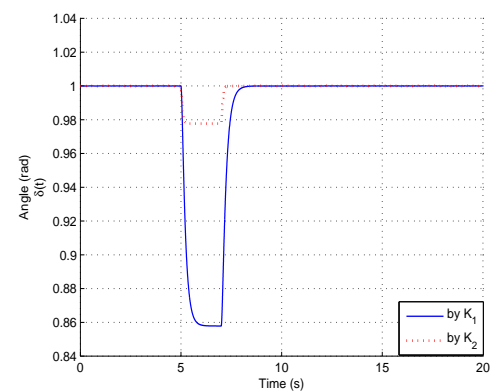

(a) Angle $x_{1}(t)=\delta$

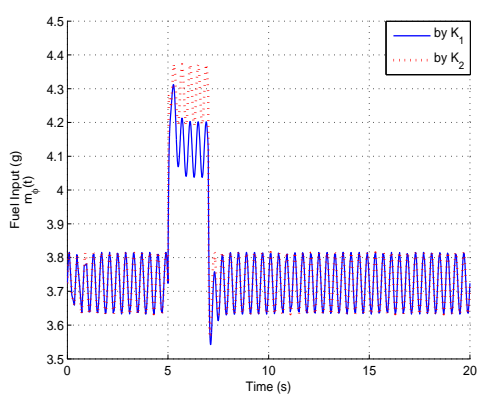

(d) Fuel mass input $u_{1}(t)=m_{\phi}$

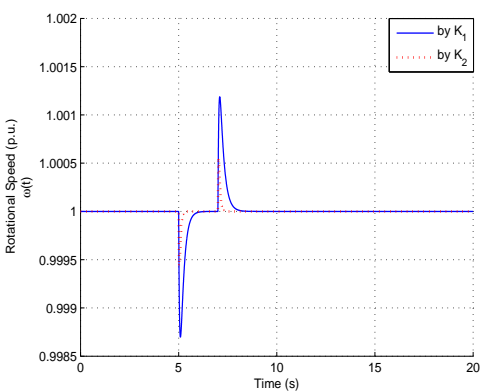

(b) Rotational speed $x_{2}(t)=\omega$

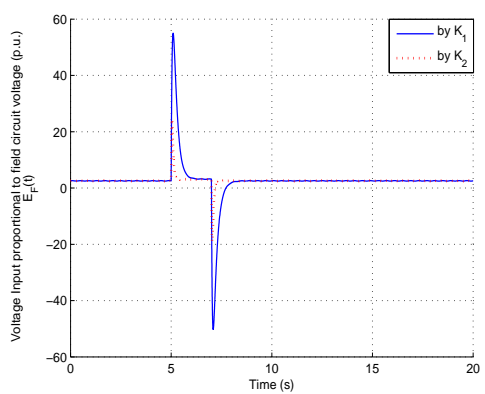

(e) Field circuit input $u_{2}(t)=E_{F}$

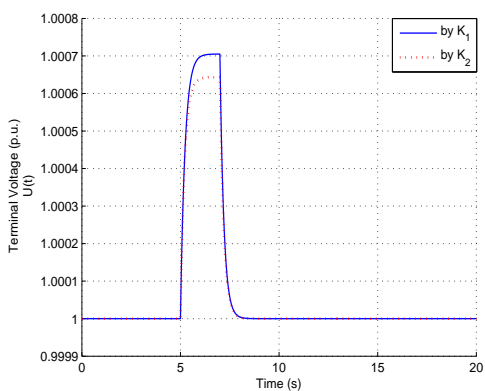

(c) Terminal Voltage $x_{3}(t)=U$

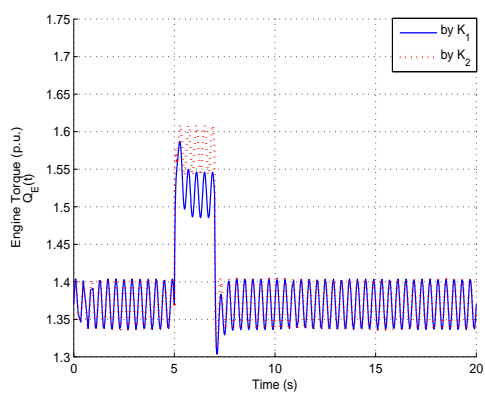

(f) Engine Torque $Q_{E}(t)$

Figure 3: Results of the simulation of the model in eq.(40) with a sudden step in stator current by gains: $\mathbf{K}_{\mathbf{1}}$ (solid) and $\mathbf{K}_{\mathbf{2}}$ (dotted)

\subsection{Time Delay}

On one hand, the model in eq.(39) is highly non-linear, and the time delay is not constant due to its dependence on the speed, as can be noted from (21). Thus, techniques like Smith predictor may not be suitable, but time delay compensation for the model in eq.(39) can be an interesting topic for future work. On the other hand, the time delay introduced by the Diesel engine is not so large, that is why it is usually neglected. What we try to show here, by simulations, is that small time delays will not affect the controller, significantly.

Generally speaking, a typical time delay of a Diesel engine is in order of milliseconds. The model in eq.(40) with the control laws in (45) was simulated one more time, with the gain $\mathbf{K}_{\mathbf{1}}$ in (53). Same parameters of the Diesel engine and synchronous machine, given in Tables 2 and 1 respectively, were used here as well. The disturbance stator current $i_{d}$ was assumed as in (51). Two time delays of $0.01 \mathrm{~s}$ and $0.04 \mathrm{~s}$ were introduced in the engine torque as in (28). The same step in the load torque was introduced, as in the previous subsection. The states trajectories are shown in Fig. 4, without the control inputs signals because they are identical to those obtained in Fig. 2 with the gain
$K_{1}$.

As can be seen in Fig. 4, the proposed controller could keep the system stable around the desired outputs with a limit cycle due to the time delay. Intuitively, the amplitude of the limit cycle increases with the time delay. The argument about the states trajectories with the increase step in the load torque, in the previous subsection, is valid here, as well. However, we need to mention here that higher gains may lead to loss of stability, especially with increasing time delays. We can motivate that by the following argument. When the gain elements corresponding to the angle and speed increase, the first input control $m_{\phi}$ plays the major role in stabilizing the system. Thus, the mass fuel input $m_{\phi}$ increases, and so does the engine torque. This magnifies the effect of the time delay since it is imposed by the engine torque. In fact, choosing the right gain is trickier now than before. Low gains of angle and speed would increase the field circuit input voltage $E_{F}$ to unreasonable levels, whereas high gains of angle and speed would not only increase the fuel mass input $m_{\phi}$, but it may also lead to losing the stability due to the time delay imposed by the engine torque. 


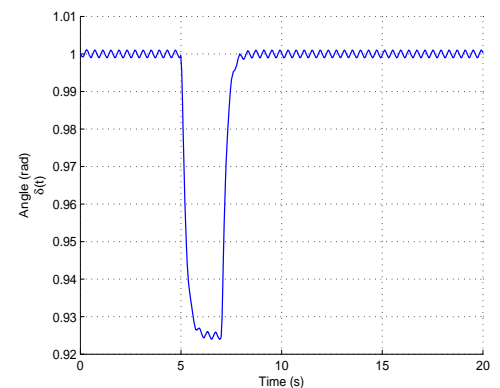

(a) Angle $x_{1}(t)=\delta, \tau=0.01 \mathrm{~s}$

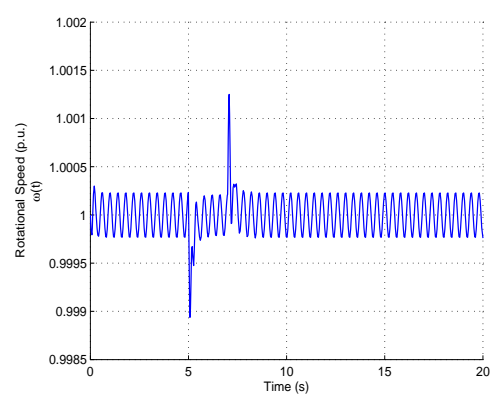

(d) Rotational speed $x_{2}(t)=\omega, \tau=$ $0.04 \mathrm{~s}$

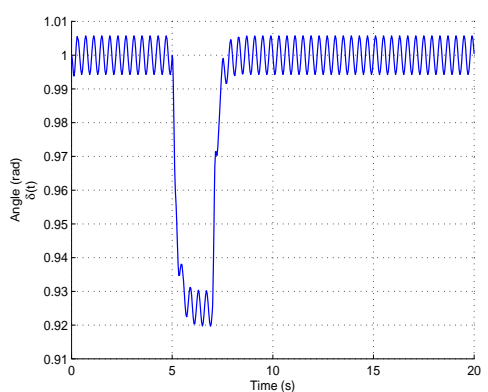

(b) Angle $x_{1}(t)=\delta, \tau=0.04 \mathrm{~s}$

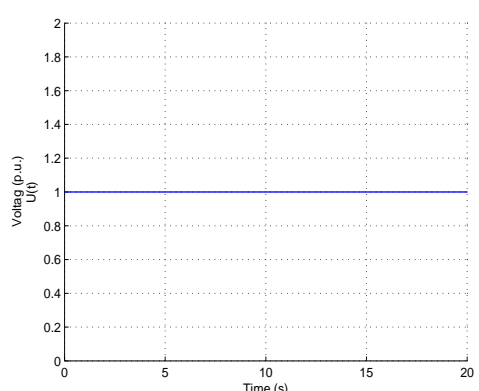

(e) Terminal Voltage $x_{3}(t)=U, \tau=$ $0.01 \mathrm{~s}$

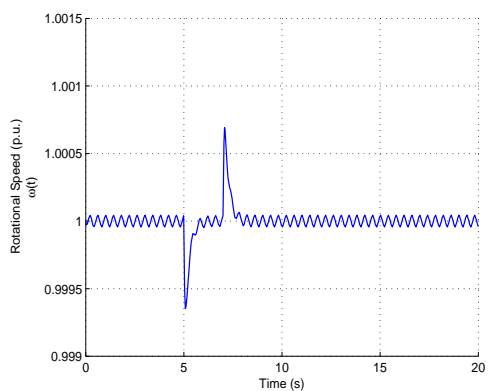

(c) Rotational speed $x_{2}(t)=\omega, \tau=$ $0.01 \mathrm{~s}$

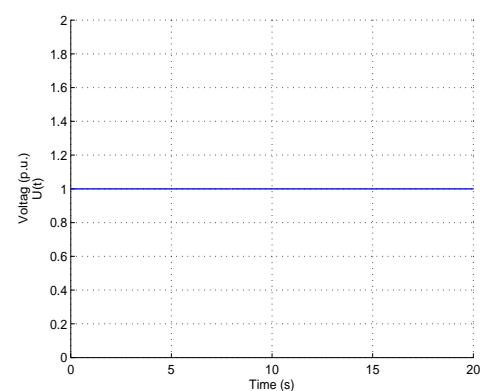

(f) Terminal Voltage $x_{3}(t)=U, \tau=$ $0.04 \mathrm{~s}$

Figure 4: Results of the simulation of the model in eq.(40) with a sudden step in the load torque for time delay of $0.01 \mathrm{~s}$ and $0.04 \mathrm{~s}$

\subsection{Uncertainty from Air/Fuel Ratio}

A combustion is called stoichiometric when "there is just enough oxygen for conversion of all the fuel into completely oxidized products, Heywood (1988)", and the air/fuel ratio corresponding to this situation is usually referred to as the stoichiometric air/fuel ratio. Generally speaking, air/fuel ratio is not desired to be too high or too low. High values of air/fuel ratio (usually called lean conditions) lead to incomplete combustion and hence decrease the efficiency, while low values (usually called rich condition) increase the emissions, Guzzella and Onder (2010). In SI engines, a three-way catalyst is used to reduce the emissions. For this catalyst to perform efficiently, the air/fuel ratio should be in a narrow band around the stoichiometric value, i.e. the relative air/fuel ratio $\lambda$ defined in (24) should be regulated to unity, Guzzella and Onder (2010).

In opposition, in CI engines like Diesel engines, the situation is more complicated. Firstly, emission reduction is taken care of by controlling the air path through the EGR, which is not necessarily at the stoichiometric air/fuel ratio. Secondly, the torque developed by the engine depends on the air/fuel ratio. Thus, the air/fuel ratio in Diesel engines is not desired to be reg- ulated around a fixed value, although this can be done through the EGR and turbocharger. For example, Alfieri et al. (2009) suggested an air/fuel ratio controller for vehicles Diesel engines around optimal set points determined from a static engine map, function of the engine speed and the mean effective pressure, obtained from steady-state measurements, Alfieri et al. (2009). Nevertheless, controllers of the modern Diesel engine usually include limiters to prevent the air/fuel ratio from reaching very high or low values, as explained above. Furthermore, the air/fuel ratio can be measured easily in modern engines by an air sensor in the exhaust manifold.

After the discussion above, one can say that the proposed model in (39) can be modified by dealing with the quantity $\Theta_{E}$ as a measurable disturbance, such as the current $i_{d}$. However, this may not be a clever choice, not only due to the time delay in the model but also because air/fuel ratio varies with fuel mass input. Thus, we propose dealing with the quantity $\Theta_{E}$ as an uncertain parameter with known nominal value, as follows:

$$
\Theta_{E}=\Theta_{E_{0}}+\delta_{\Theta_{E}},
$$




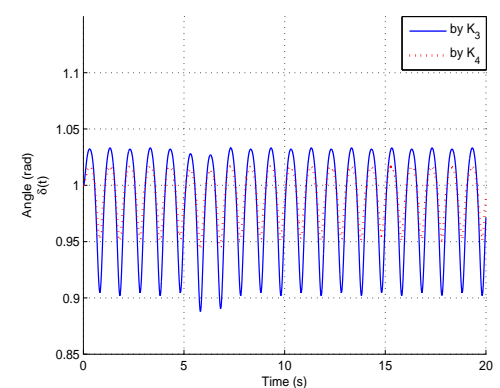

(a) Angle $x_{1}(t)=\delta$

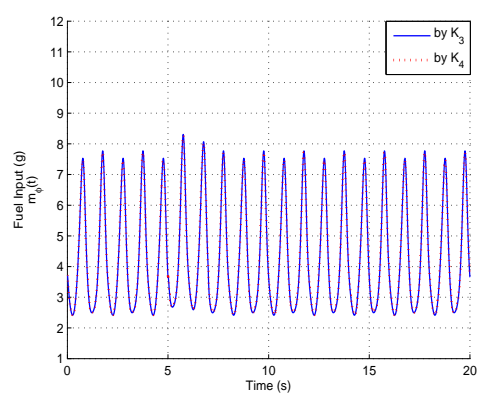

(d) Fuel mass input $u_{1}(t)=m_{\phi}$

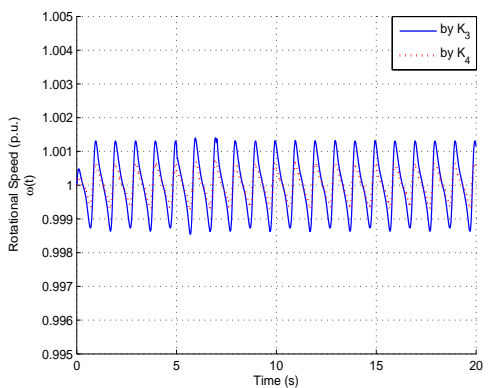

(b) Rotational speed $x_{2}(t)=\omega$

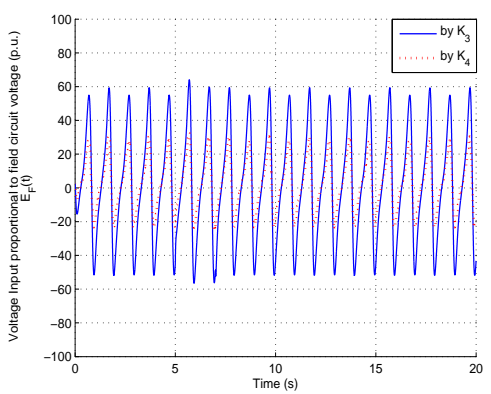

(e) Field circuit input $u_{2}(t)=E_{F}$

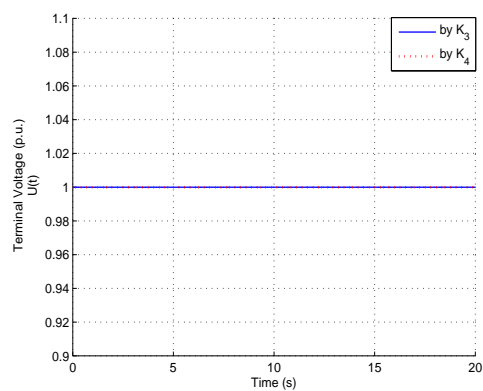

(c) Terminal Voltage $x_{3}(t)=U$

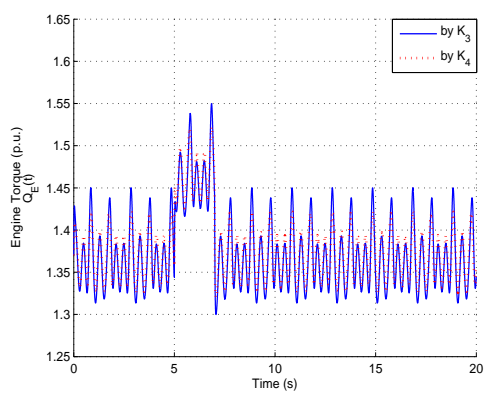

(f) Engine Torque $Q_{E}(t)$

Figure 5: Results of the simulation of the model in eq.(40) with a sudden step in the load torque by the gains: $\mathbf{K}_{\mathbf{3}}$ (solid) and $\mathbf{K}_{\mathbf{4}}$ (dotted) with $\Theta_{E}$ modelled as in (54)

where $\Theta_{E_{0}}$ is the measurable nominal value, and $\delta_{\Theta_{E}}$ is unmeasurable. Several authors in the field of robust control have suggested techniques to treat the problem of the uncertain parameters. One of the most attractive techniques in the literature to deal with uncertain parameters with feedback linearisation is the one proposed in Kolavennu et al. (2001) for square MIMO systems, which was expanded later in Palanki et al. (2003) for non-square models. Kolavennu et al. (2001) suggested expanding the control law, found by feedback linearisation, by Taylor's series around the nominal values of the uncertain parameters. Then, instead of using pole placement to find the gain, the authors suggested using $H_{2} / H_{\infty}$ synthesis and they proved, based on the work by Khargonekar and Rotea (1991), that the solution of the mixed $H_{2} / H_{\infty}$ synthesis problem stabilizes the model with the uncertain parameters, Kolavennu et al. (2001). In spite of the elegance of their proposal, the authors did not provide enough information on choosing the weighting matrices, which makes the proposal cumbersome to apply, at least in our model. Anyway, we show in the sequel, by simulations, that modelling $\Theta_{E}$ as in (54) does not influence the proposed controller. The model in eq.(40) was simulated one more time, neglecting the time delay. Same parameters of the Diesel engine and synchronous machine, given in Tables 2 and 1 respectively, were used here as well. The disturbance stator current $i_{d}$ was assumed as in (51). The same step in the load torque was introduced, as in the previous subsections. The nominal value $\Theta_{E_{0}}$ was assumed 0.4 , and the variation $\delta_{\Theta_{E}}$ was assumed a sinusoidal function as $0.2 \sin (2 \pi t)$. In order to mimic the situation, that the nominal value of the uncertain parameter is fed forward to the controller and variation is not, the control laws in (45) were calculated at the nominal value $\Theta_{E_{0}}$. The states trajectories are shown in Fig. 5, with the control inputs signals.

Our experiments emphasized the fact we showed previously, that low gains make the second control input $E_{F}$ reach high levels. The uncertainty make $E_{F}$ increase even more. Hence, we had to increase the gains. Two gains are shown in the results depicted in Fig. 5, as follows:

$$
\begin{array}{lrl}
\text { at poles } & {[-5-20-50] \longrightarrow \mathbf{K}_{\mathbf{3}}=\left[\begin{array}{ccc}
1000 & 70 & 0 \\
0 & 0 & 5
\end{array}\right]} \\
\text { at poles } \quad[-5-20-100] \longrightarrow \mathbf{K}_{\mathbf{4}}=\left[\begin{array}{ccc}
2000 & 120 & 0 \\
0 & 0 & 5
\end{array}\right]
\end{array}
$$

Due to the uncertainty, limit cycles are noticed again in the trajectory of the angle and the speed. The am- 
plitude of this limit cycle in the speed trajectory is less than $0.1 \%$, which could be very conservative because usually in power systems variation of frequency less than $1 \%$ are acceptable. For the angle trajectory, the situation is worse, especially when we note that the setpoint to be tracked is shifted a little bit below 1 . To sum up, we can say that the controller could keep the speed oscillating around the setpoint with a small amplitude, even when $50 \%$ uncertainty in $\Theta_{E}$ is applied to the model.

\subsection{Uncertainty from Air/Fuel Ratio with Time Delay}

We show finally the results of our simulations when the uncertainty is applied to the system and the time delay is taken in consideration. From the parameters in Table 2, and by using (21), we can say that for this particular Diesel engine the time delay does not exceed $0.01 \mathrm{~s}$. Thus, we simulated the model again, with same step in the load torque, but this time with $0.01 \mathrm{~s}$ time delay in the torque model. $\Theta_{E}$ was modelled as in the previous subsection. The same gains $\mathbf{K}_{\mathbf{3}}$ and $\mathbf{K}_{\mathbf{4}}$ in (55) were used here, as well. The results are shown in Fig. 6.

We can note that the results shown in Fig. 6 are indifferent from those shown in Fig. 5, except for some disturbance in the angle and speed trajectories when the sudden step in the load torque was first introduced at $t=5 \mathrm{~s}$ and when it was removed at $t=7 \mathrm{~s}$, when the higher gain $\mathbf{K}_{\mathbf{4}}$ was used. Of course, this is expected, since increasing the gain manifests the dependence on the first control input $m_{\phi}$ which, in turn, increases the effect of the time delay, as explained before.

\section{Conclusion}

In this paper a control-oriented model of the Genset, that comprises a Diesel engine and a synchronous generator, was proposed. The air/fuel ratio was modelled as an uncertain parameter of measurable nominal value, and unmeasurable variation. A controller to regulate the shaft speed and the terminal voltage, simultaneously, was designed by using feedback linearisation. The controller consists of two control inputs, the fuel mass input and the field circuit voltage. Simulations of the proposed model with the proposed control laws were provided. The simulations show the following:

1. The suggested controller uses both control inputs to stabilize the system, simultaneously, in contrast to the ordinary PID where no direct communication exists between the speed governor and the AVR.
2. Choosing the gains of the control laws is a trade-off between reducing the fuel consumption and avoiding high values of field circuit voltage. Increasing the gain corresponding to the fuel input increases the fuel consumption, and reduces the dependence on the field circuit voltage, and vice versa.

3. Since the time delay in the engine torque model is not so large, with careful choosing of the gains, the proposed controller can still stabilize the states with limit cycles of very small amplitude.

4. The uncertainty of the air/fuel ratio increases the amplitude of the limit cycles reached by the states, and may change the desired set points to be tracked, especially for the angle state trajectory. However, the set point of the speed is not changed, and the amplitude of its limit cycle can be minimized with astute tuning of gains. Thus, the proposed controller can be considered robust to the uncertainties.

\section{Acknowledgements}

The authors would like to thank the Norwegian Research Council, SINTEF Fisheries and Aquaculture, and Rolls-Royce for sponsoring this work through the ImproVEDO-project.

\section{References}

Alfieri, E., Amstutz, A., and Guzzella, L. Gainscheduled model-based feedback control of the air/fuel ratio in diesel engines. Control Engineering Practic, 2009. (17):1417-1425. doi:10.1016/j.conengprac.2008.12.008.

Fossen, T. I. Guidance and Control of Ocean Vehicles. John Wiley \& Sons, 1994.

Goh, K. B., Spurgeon, S. K., and Jones, N. B. Higher-order sliding mode control of a diesel generator set. Proceedings of the Institution of $\mathrm{Me}$ chanical Engineers, Part I: Journal of Systems and Control Engineering, 2003. 217:229-241. doi:10.1177/095965180321700305.

Guzzella, L. and Amstutz, A. Control of diesel engines. IEEE Control Systems, 1998. 18:53-71. doi:10.1109/37.722253.

Guzzella, L. and Onder, C. H. Introduction to Modeling and Control of Internal Combustion Engine Systems. Springer, 2nd edition, 2010. 


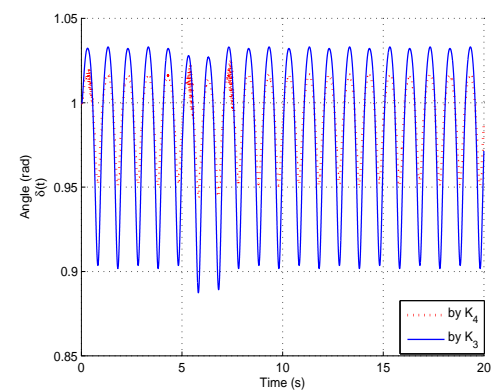

(a) Angle $x_{1}(t)=\delta$

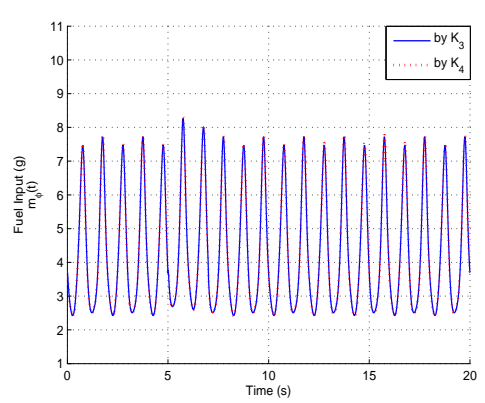

(d) Fuel mass input $u_{1}(t)=m_{\phi}$

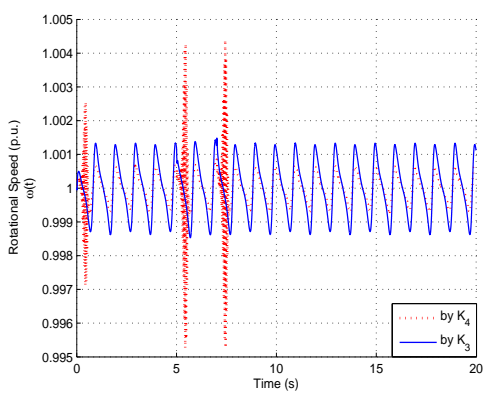

(b) Rotational speed $x_{2}(t)=\omega$

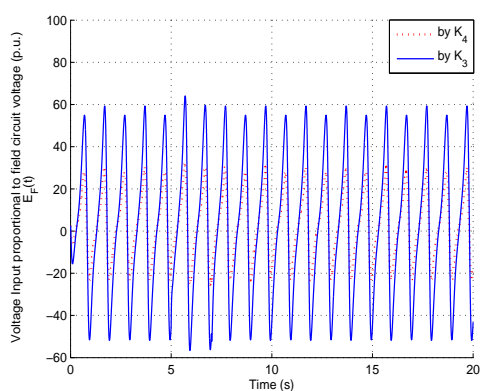

(e) Field circuit input $u_{2}(t)=E_{F}$

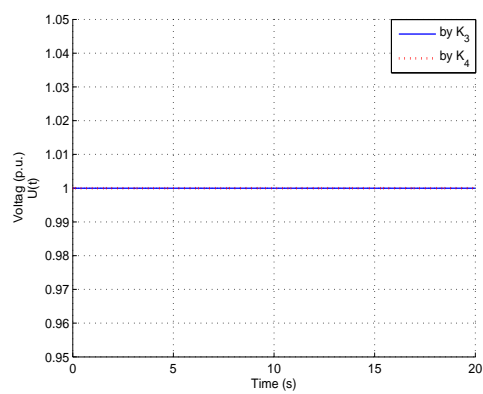

(c) Terminal Voltage $x_{3}(t)=U$

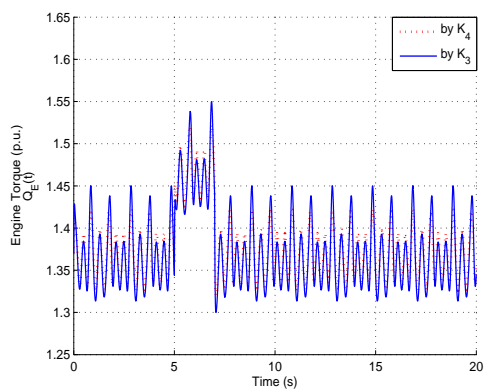

(f) Engine Torque $Q_{E}(t)$

Figure 6: Results of the simulation of the model in eq.(40) with a sudden step in the load torque by the gains: $\mathbf{K}_{\mathbf{3}}$ (solid) and $\mathbf{K}_{\mathbf{4}}$ (dotted) with $\Theta_{E}$ modelled as uncertain parameter and time delay of $0.01 \mathbf{s}$

Hansen, J. F. Modelling and Control of Marine Power Systems. Ph.D. thesis, Norwegian University of Science and Technology, 2000.

Hansen, J. F., dnanes, A. K. A., and Fossen, T. I. Mathematical modeling of diesel-electric propulsion systems for marine vessels. Mathematical and Computer Modeling of Dynamical Systems, 2001. 7:323355. doi:10.1076/mcmd.7.3.323.3641.

Heywood, J. B. Internal Combustion Engine Fundamentals. McGraw-Hill, 1988.

Huang, M. L. Robust control research of chaos phenomenon for diesel-generator set on parallel connection. Applications of Nonlinear Control, Intec., 2012. doi: $10.5772 / 36826$.

Isidori, A. Nonlinear Control Systems. Springer, 2nd edition, 1989.

Jensen, J. P., Kristensen, A. F., Sorenson, S. C., Houbak, N., and Hendricks, E. Mean value modeling of a small turbocharged diesel engine. Society of Automotive Engineers (SAE), 1991. (910070). doi:10.4271/910070.

Kao, M. and Moskwa, J. J. Engine load and equivalence ratio estimation for control and diagnostics via nonlinear sliding observer. In Proceedings of the American Control Conference. pages 1574-1578, 1994. doi:10.1109/ACC.1994.752334.

Khargonekar, P. P. and Rotea, M. A. Mixed $h_{2} / h_{\infty}$ control: A convex optimization approach. IEEE Transactions on Automatic Control, 1991. 36(7):824-837. doi:10.1109/9.85062.

Kolavennu, S. N., Palanki, S., and Cockburn, J. C. Robust controller design for multi-variable nonlinear systems via multi-model $H_{2} / H_{\infty}$ synthesis. Chemical Engineering Science, 2001. 56:4339-4349. doi:10.1016/S0009-2509(01)00034-3.

Kundur, P. Power System Stability. McGraw-Hill, Inc., 1994.

Machowski, J., Bialek, J. W., and Bumby, J. R. Power System Dynamics: Stability and Control. John Wiley \& Sons, 2nd edition, 2008.

McCowan, D. J., Morrow, D. J., and McArdle, M. A digital pid speed controller for a diesel generating set. In IEEE Power Engineering Society General Meeting, volume 3. pages 1472-1477, 2003. doi:10.1109/PES.2003.1267371. 
McGowan, D. J., Morrow, D. J., and Fox, B. Integrated governor control for a diesel-generating set. IEEE Transactions on Energy Conversion, 2006. 21(2):476-483. doi:10.1109/TEC.2006.874247.

Palanki, S., Cockburn, J. C., and Kolavennu, S. N. Robust state feedback synthesis for control of non-square multivariable nonlinear systems. Journal of Process Control, 2003. 13:623-631. doi:10.1016/S0959-1524(02)00098-7.

Pivano, L., Johansen, T. A., Smogeli, O. N., and Fossen, T. I. Nonlinear thrust controller for marine propellers in four-quadrant operations. In Proceedings of the American Control Conference. pages 900-905, 2007. doi:10.1109/ACC.2007.4282514.
Rahman, M. A., Osheiba, A. M., Radwan, T. S., and Abdin, E. S. Modelling and controller design of an isolated diesel engine permanent magnet synchronous generator. IEEE Transactions on Energy Conversion, $1996 . \quad 11(2): 324-330$. doi:10.1109/60.507185.

Tuffaha, M. and Gravdahl, J. T. Modeling and control of a marine diesel engine driving a synchronous machine and a propeller. In 2014 IEEE International conference on Control Applications (CCA) Part of 2014 IEEE Multi-conference on Systems and Control. Antibes-France, pages 897-904, 2014. doi:10.1109/CCA.2014.6981450. 